\title{
Les déterminants d'arguments matrices face aux relatives extraposées
}

\author{
Lucien Kupferman \\ Université de Tel Aviv (Israël) \\ kupferma@post.tau.ac.il
}

\begin{abstract}
Résumé. On veut décrire et analyser les propriétés interprétatives qui particularisent les propositions relatives extraposées à partir d'arguments sujets définis (dans le texte: DefExtrap). Dans un premier temps, on les distingue des relatives extraites d'arguments sujets indéfinis (dans le texte: IndefExtrap). Ensuite, on observe que le prédicat enchâssé de DefExtrp doit nécessairement être événementiel. Celui de la matrice est, dans une première description, limité à la dénotation d'espaces, qui localiseraient l'argument. Et puis, une analyse plus poussée montre que ce prédicat décrit en fait des états transitoires de l'entité prédiquée, et il est chargé de la sorte d'introduire l'événement enchâssé, qui représente le focus de l'information véhiculée par la phrase. Cette analyse des constructions DefExtrap, comme des représentations d'états épisodiques introduisant des descriptions d'événements, permet d'explique la constatation qui est faite que sont interdites les matrices niée, génériques, et hypothétiques-irréelles. Deux questions ne sont pas traitées dans la communication. Tout d'abord, l'explication de la limitation des possibilités du relatif-tête à la forme qui, c'est-à-dire à un élément dont la source serait strictement la position sujet de l'enchâssée. Ce développement serait d'ordre syntaxique, ce qui excéderait le programme de l'atelier. Ensuite, une explication de la nécessaire (quasi-) simultanéité des intervalles de temps des dénotations des deux propositions impliquées. Enfin, la parenté patente entre certaines des propriétés de DefExtrap et des relatives prédicatives reste à élucider
\end{abstract}

\begin{abstract}
Matrix-argument determiners in front of extraposed relative clauses. The following study is aimed at a description and an analysis of the properties of that subset of relative clauses as extraposed from definite external arguments (thereafter : DefExtrap). At a first stage, they are differentiated from indefinite extraposed-relative clauses associated with external arguments (thereafter: IndefExtrap). At a second stage, it is pointed out that embedded clause in DefExtrep necessarily have to denote event-oriented predicates. In a first descriptive level, the matrix-predicates appear as space denotations which function as clausal argument locaters. Then, a thorough analysis shows that in fact these predicates describe transitory states of the predicated entity, so that their basic task is to introduce the embedded event, which embodies the focus of the sentential information. This analysis of the DefExtrap constructions, as representations of episodic states that introduce descriptions of events, fits with the intended explanation of various data as disallowance of negative, generic, irrealishypothetical matrix clauses. Two issues are not addressed in the talk. First, the explanation of the restriction laid on the head-relatives to the only item qui, i.e. to an item which the source of would strictly be the embedded subject. This development would be purely a syntactic one, and it would excess the framework of the panel. And then, an explanation of the (quasi-) coincidence between the time-intervals of both clause denotations is lacking. At last, some properties patently common to DefExtrap and to the set of predicative-relative clauses wait for forthcoming clarification.
\end{abstract}




\section{Introduction}

Les relatives extraposées sont celles qui, modifiant les SN arguments des propositions matrices, se retrouvent à la suite du SV de ces dernières. En conséquence, et contrairement aux relatives standard, ces propositions ne sont pas des adjoints des SN qu'elles modifient. Cette construction relève sans nul doute du registre dit «de la conversation soignée », et les contraintes de connexion séquentielle qui jouent dans le parler habituel en sont, en partie, absentes. On trouve à son sujet des observations utiles chez Rothenberg 1978, Prebensen 1982, qu’on distingue ici des relatives prédicatives, v. un échantillon de la riche masse d'études faites à leur propos dans la bibliographie.

Dans le cas standard, l’argument modifié par une relative extraposée est quantifié (v. Kupferman 2014) :

(1) $(*$ le+*mon+*ce+un+quelque) chat venait de sauter qui se préparait à attaquer (*le+un) corbeau est passé qui croassait dans un vacarme assourdissant (*les+des) passants se sont attroupés qui ont regardé le cracheur de flammes

Les déterminants définis sont rejetés de ce type général d’environnement, de sorte que le schéma qui résulte de cette propriété a la forme (où : $\arg _{\text {quant }}=$ argument quantifié) :

(2) IndefExtrap :

$\mathrm{X}$-arg quant $\mathrm{Y}$ - SV-Relative- Z.

La classe des constructions qui obéissent à (2) reçoit ici le sigle IndefExtrap, qui cible le fait qu'un argument matrice est quantifié (« indéfini ») s’il se combine à une relative extraposée.

Et pourtant, un énoncé comme (3) où l'argument matrice est défini ne pose pas de difficulté à un auditeur (adapté d’une remarque de Francis Corblin) :

(3) le chat était là qui miaulait à te fendre le cœur

De plus, l'énoncé (4) peut se substituer à cette séquence sans qu’on puisse observer de différences entre les valeurs de vérité de ces phrases bi-propositionnelles :

(4) le chat était là et miaulait à te fendre le cœur

Face au schéma (2) de IndefExtrap, des exemples comme (3) répondent au schéma (5), où Def=défini.

(5) DefExtrap :

$\mathrm{X}-\arg _{\text {def }}-\mathrm{Y}-\mathrm{SV}$-relative-Z

(1) et (3) demandent qu'on circonscrive les raisons du contraste qui s'établit entre les types (2) et (5). Cette question fait l'objet de l'étude qui suit. On se demande en particulier pourquoi l'exclusion des déterminants définis est levée dans le cas de (3/5), celui-ci étant caractérisé, en premier lieu comme on le verra, par la présence de prédicats matrices spatiaux.

On ouvre le débat par la définition des propriétés de DefExtrap. On constate d’abord, section 2, que ce schéma assigne au prédicat matrice une fonction de localisation dans l'espace de l'argument auquel il est appliqué. La section 3 examine de plus près la nature de ce prédicat, pour arriver à une généralisation qui remplace la catégorie «spatial » de ce dernier par celle d'état transitoire qui la transcende.

Une situation où les schémas (2) et (5) impliquent tous deux des relatives qui ne se trouvent pas dans des positions adjointes aux arguments propositionnels qu'elles modifient amène à se demander si le premier est un sous-type du second. La réponse à cette question conduit à une description, dans la section 2, des propriétés singulières de DefExtrap face à IndefExtrap. 


\section{Différences entre DefExtrap et IndefExtrap}

DefExtrap et IndefExtap divergent par la limitation, inconnue du second, de la tête relative du premier à la forme qui sujet, section 2.1. Ils diffèrent aussi, section 2.2, par la relation qu'ils établissent entre les époques des deux propositions, concomitance pour le premier, liberté pour le second. Enfin, section 3, le premier exige la présence d'un prédicat de localisation, et donne toute latitude au second. Il ressort de ces comparaisons que IndefExtrap produit une indétermination a priori plus étendue que son congénère DefExtrap. Les particularités de DefExtrap présentent une certaine similitude avec celles des relatives prédicatives, v. sur ces relatives Kayne 1977, Muller 2011, Kleiber 2000, Guasiti 1998, Cecchotti 2015, et autres - v. Bibliographie.

\subsection{DefExtrap, contrairement à IndefExtrap, n'accepte, pour la tête relative, que la forme qui (sujet).}

Dans les relatives extraposées associées à des DP sujets définis, les relatifs têtes récusent toute source qui n’est pas la position sujet. Cette propriété réunit par ailleurs DefExtraqp et les structures à relative prédicative (v. pour ce dernier type les références ci-dessus) :

(6) a. la voisine était sur le palier (qui faisait son yoga+*que Léa observait+*à qui Sam racontait des boniments)

$$
=\text { DefExtrap }
$$

b. certains touristes étaient sur le site (qui photographiaient les deux colonnes +qu'un guide peinait à rassembler+à qui un guide local donnaient des explications incompréhensibles)

$$
=\text { IndefExtrap }
$$

c. Max (a trouvé sa voisine+l’a trouvée) sur le palier (qui faisait son yoga+*que

Léa observait+*à qui Sam racontait des boniments)

= relative prédicative enchâssée

La différence notée ici entre les deux sortes de relatives extraposées témoigne d'une discordance importante entre elles. L'explication de ce contraste exige une prise en compte des configurations syntaxiques exclue de cette étude pour des raisons de place et de temps.

\subsection{Dans DefExtrap, simultanéité des intervalles de temps de la matrice et de la relative extraposée.}

Comme dans les coordonnées de (4), DefExtrap véhicule une simultanéité nécessaire des dénotations des deux propositions. (8ai) devient ainsi irrecevable. Cette impossibilité ne peut pas être imputée à la différence des temps sémantiques en tant que telle, v. (8aii) où la relative n’est pas extraposée, mais seulement adjointe au SN argument qu'elle modifie. Si, par contre, les éventualités sont situées dans des intervalles de temps contemporains, la propriété 2.2 légitime les énoncés de (8b-d).

(8) a. i. *le chat était là qui (avait miaulé+miaulerait) après Ida

ii. le chat qui (avait miaulé+miaulerait) après Ida était là

b. et maintenant le chat est là, qui (miaule+*a miaulé) après Ida

c. le chat a soudain été là, qui (s’est mis à miauler+*avait miaulé) à te fendre le cœur

Cette contrainte ne s’applique pas à IndefExtrap : 
(9) des manifestants affluaient, qui (étaient arrivés+arrivaient) par autocars entiers des pruniers étaient couchés sur le sol, qui seront enlevés dès que possible un chat a sauté, qui (avait déjà griffé l’enfant

La simultanéité des épisodes des deux propositions de DefExtrap, qui tranche sur la liberté des temps sémantiques de IndefExtrep, indique que la relation de ces deux propositions est bien plus forte. L’occurrence d'une relative extraposée dépend dans DefExtrap de cette simultanéité.

\subsection{DefExtrap exige une matrice copulative}

Contrairement à IndefExtrap, DefExtrap ne supporte pas les matrices événementielles. Bien plus, la classe des prédicats admis par le premier ne comprend pas seulement les états transitoires, mais aussi des événements qui repèrent des sous-ensembles d'entités dans un monde instanciés par l'argument propositionnel (v. pour une discussion récente Kupferman 2014). DefExtrap demande, en revanche, que ces sous-ensembles soient articulés autour d'un verbe copule.

\subsubsection{DetEXtrap récuse les prédicats événementiels}

On a montré dans Kupferman 2014 que IndefExtrap exige de son argument matrice qu’il soit quantifié faiblement. Cette obligation a pour corollaire nécessaire que la construction demande des prédicats matrices d'événements ou d'états transitoires. Or, l'environnement des événements est indésirable pour DefExtrap. Soit, par exemple :

(10) (*la+une) porte claquait qui demandait à être refermée (*la+quelque) marche manquait qui risquait de produire des accidents (*les+de) nouvelles classes se sont ouvertes qui doivent accueillir une vingtaine d'élèves chacune

Les exemples (10) illustrent le fait que DefExtrap, contrairement à son cousin IndefExtrap, n’admet pas de prédicats événementiels.

\subsubsection{La matrice de DefExtap est copulative}

Une matrice qui comporte un argument défini modifié par une relative extraposée doit être copulative.

(11) a. Le chat (*ronronnait+était) dans la tombe), qui regardait Quentin

b. Les touristes (*photographiaient+étaient) sur le site, qui, visiblement, s’impatientaient déjà

Cette nécessité d’une copule indique que le prédicat matrice de DefExtrap n’est pas verbal, mais est instancié - au moins -par l'adverbial de lecture spatiale. On voit à la section 3.1 que ce même prédicat peut aussi être de nature adjectivale. Il installe la dénotation de l'argument auquel il est attribué dans une situation où elle est décrite comme une cause d'événement ; elle est décrite donc, non pas tant par le prédicat matrice que, au travers du relatif sujet qui, par le prédicat de la relative extraposée.

On vient de noter que les relatives extraposées de DefExtrap doivent être, à l'encontre de leur matrice, de nature événementielle. Cette particularité est illustrée ici :

(12) (*le+un) chat était dans la tombe, qui appartenait à Quentin (*le+un) guéridon est dans le couloir, qui gêne le passage 
Les prédicats des relatives extraposées de (12) dénotent des propriétés, elles sont refusées. Les options quantifiées sur les arguments matrices n’opposent aucune difficulté aux prédicats enchâssés qui dénotent des propriétés : on note à nouveau que IndefExtrap dispose d'une marge de manœuvre refusée à DefExtrap. On observe encore que les déterminants sur les arguments matrices exercent leurs effets sur les enchâssées. Le relatif qui semble transparent à cet égard.

\subsubsection{Le prédicat matrice spatial de DefExtrap localise son argument}

Les catégories des prédicats de (11a)-(11b) sont instanciées par des expressions spatiales. Cette propriété peut encore s’illustrer par (13) :

(13) Le chat était à (a.*Ida+ b. la cuisine), qui miaulait sans arrêt La machine est à (a.*circuits intégrés+b. l'atelier) qui attend toujours un technicien Le patient est au (a.*Dr Paule+b. bloc opératoire) qui subit des soins extrêmement intensifs

La nature des prédicats matrices en à dans les deux options qui contrastent à chaque fois Possession/Localisation, Manière/Localisation, Destinataire/Localisation - entraîne des résultats différents. L'option qui échappe au crash est toujours celle de la lecture qui localise l'argument dans un monde. Observons aussi, ce qui aura une importance centrale dans la section 3 qui suit que les options a renvoient toutes à des propriétés, et les options b à des localisations précaires.

La section 2.2 est parvenue aux résultats majeurs suivants : dans une construction IndefExtrap, la matrice dispose d’une large variété de prédicats qui peuvent dénoter, soit des états, soit des événements, le prédicat enchâssé peut renvoyer à des états, des propriétés, à des événements. La construction est à tous ces égards largement non contrainte. Sous DefExtrap, le prédicat matrice définit l'espace où se déroule un événement décrit par la relative extraposée et prédiqué de l'argument matrice.

\section{Les prédicats matrices de DefExtrap : analyse}

A la réflexion, et dans son principe-même, la description faite ci-dessus nous laisse sur notre faim. Quel peut bien être en effet le lien entre les trois paramètres : relative extraposée, argument matrice défini, et prédicat matrice spatial ? Pourquoi la nature de ce prédicat, somme toute très particulière une dénotation d'espace - est-elle une condition de l'occurrence des constructions DefExtrap ?

Cette section 3 veut répondre à la question ainsi posée. Elle part d'une analyse des prédicats spatiaux engagés dans DefExtrap, qui montre que la propriété «spatial » gagne dans ce type de constructions à être surclassée. Cette analyse fait profiter ladite propriété d’une généralisation vraie et significative : les prédicats matrices de DefExtrap sont des expressions qui dénotent des états transitoires attribués a la dénotation de l'argument (section 3.1). L'analyse est ensuite vérifiée au moyen de trois réactifs qui mettent en jeu des environnements d'abord négatifs (section 3.2), puis contrefactuels (section 3.3), et enfin génériques (section 3.4).

\subsection{Une installation précaire de la dénotation de l'argument matrice de DefExtrap}

Le type (11a-b) n’est qu'un sous-cas d’une catégorie plus large. Au premier abord, c'est l'inverse qui semblerait vrai. Dans ces exemples et dans ceux du même genre qui suivent, la localisation, qui s'établit dans un monde pour des arguments matrices de DefExtrap, installe une relation précaire. Les relatives extraposées refusent toute combinaison avec des prédi-cats matrices, spatiaux ou autres, qui dénoteraient des états stables, virtuellement perma- nents. L’occurrence de ces prédicats paraît plus 
contrainte que ne l'énonçait la propriété <spatial>. L’ensemble des prédicats pertinents inclut également des adjectifs non spatiaux, à la condition qu’ils renvoient à des états précaires. La comparaison des exemples i acceptés et ii récusés révèle qu'une analyse plus poussée de DefExtrap exige que la proprié-té <spatial> des prédicats matrices de DefExtrap soit remplacée par celle de $<$ transitoire $>$.

(13) a. Le chat était (I. à cet instant +ii. *à demeure) chez elle, qui miaulait à cœur fendre

b. (i. la voiture+ii.*la piazza) était devant le garage, qui allait être nettoyée par notre équipe

c. Le prunier est (i. couché +ii.*planté) dans notre jardin), qui attend d’être enlevé

d. i. Le coursier est à la porte (Ø+, qui attend une réponse de Léa)

ii. La population la plus démunie est dans notre quartier (Ø+*, qui attendent des réponses de l’Office départemental des HLM)

Ces contextes font contraster, dans les prédicats matrices, des états transitoires, autrement dit conçus comme des phases des entités arguments, ce sont las exemples i. de (13)), avec des états envisagés comme des états permanents, c’est-à-dire des propriétés d'entités, ce sont les données ii. Les options i sont toujours autorisées dans ces constructions DefExtrap, et les options ii refusées.

Ce n'est donc pas le fait que la dénotation de l'argument matrice soit positionnée dans un monde qui est décisif dans la formation de DefExtrap, mais le fait que cette situation soit transitoire, épisodique. Cette stipulation d'un positionnement est même en principe superflue. La condition indispensable à DefExtrap est seulement l'occurrence d'une expression prédicative de niveau de phase (=dénotant un état précaire). Cette généralisation est satisfaite, non seulement par les adverbiaux spatiaux i de (a-d), mais aussi par des adjectifs de nature transitoire.

On doit vérifier par la négative, en dehors des environnements récusés dans la section 2, le bien fondé de la généralisation figurant dans le titre de cette section 3.1.

\subsection{Une matrice de DefExtap ne peut pas être niée}

\subsubsection{Caractère crucial de la propriété « transitoire »}

Quelle est la raison d'être de cette contrainte, qui restreint à ceux qui sont rassemblés par la propriété «transitoire» la classe des prédicats matrices possibles de DefExtrap, et écarte d'emblée toute autre catégorie?

\subsubsection{Complémentarité des propriétés des deux propositions de DefExtrap}

La réponse est à chercher dans la complémentarité des propriétés des deux propositions de DefExtrap. Cette complémentarité résulte du fait que la matrice a pour rôle strict d'intro-duire l'événement dénoté par la relative extraposée. En particulier, v. section 2.2.1, l'inter-valle de temps où prend place l'état dénoté par la matrice doit recouvrir celui où se déroule l’événement de la relative extraposée. Ce qui peut aussi s'énoncer ainsi : l'ensemble des instants de la seconde dénotation doit fonctionner comme un sous-ensemble de l'ensemble des instants de la première, ou être égale à lui :

L’identité de référence des deux arguments des propositions, le second étant instancié par la forme qui, indique que c'est à la matrice qu'il appartient d'introduire l'information concer-nant la nature de l’argument sujet enchâssé. 
Et la coïncidence entre les deux intervalles de temps induit que c'est la matrice qui installe celui de l'enchâssée. Le fait que le verbe matrice soit nécessairement une copule, forme vide de contenu sémantique, confirme ce diagnostic. Le caractère événementiel de la relative extraposée entraîne pour le prédicat matrice l’obligation d'une dénotation de type transitoire.

La nature précaire des prédicats matrice est ainsi une partie intégrante des propriétés de complémentarité nécessaire qui définissent la matrice et la relative de DefExtrap.

\subsubsection{Ici aussi : prédicats de niveau de phase et négation}

Comme on sait, des arguments à quantifieur faible ne peuvent pas figurer sous des opérateurs négatifs. Ainsi, les options négatives ii des exemples (14) ne parviennent pas à délimiter le domaine de quantification demandé par l’argument quantifié faiblement de la proposition :

(14) une idée folle (i. vient+ii.*ne vient pas) de lui passer par la tête des passants (i. se sont+ii.*ne se sont pas) attroupés autour de Max des touristes (i. ont+ii.*n'ont pas) pris de photos aujourd'hui quelque prunier (i.est+ii.*n'est pas) couché par terre

L'existence des sous-ensembles décrits par les arguments matrices des options ii de (14) se verrait installée, d'une part, dans un monde par les quantifieurs et serait d'autre part niée par l'opérateur inclus dans ces matrices. La contradiction entre les deux visées est insoutenable, et les propositions crashent.

DefExtrap semblerait répondre à ce réactif de la négation appliquée à la quantification faible. Ce qui ne peut pas être, puisque les prédicats matrice des relatives extraposées des exemples ii de (15) sont attribués à des SN définis, c’est-à-dire à des sous-ensembles installés préalablement à la situation.

(15) le chat n’est pas dans le couloir (i. Ø +ii. *, qui miaule à te fendre le cœur)

Le conflit que la négation met en jeu dans DefExtrap l'oppose, non pas à un argument quan-tifié, mais à un prédicat de niveau de phase. Ainsi, dans (15), l’opérateur négatif annule l'état voulu par être dans la cour. Mais cet état de la dénotation de l'argument a, par ailleurs, pour fonction d'introduire l'évènement, tâche qu’il ne peut pas exécuter.

\subsection{DefExtrap ne peut pas renvoyer à des mondes contrefactuels}

Les états transitoires des matrices de DefExtrap contrefactuel dénoteraient des non-événements, et contreviendraient à la fonction assignée à ces constructions.

(16) si le chat était maintenant là ( ${ }^{* *}$ qui se préparerait à attaquer), Dan prendrait ses précautions

v. si le chat était maintenant là et qu'il se préparerait à attaquer, Dan prendrait ses précautions = pas de relative extraposée

Les énoncés contrefactuels présupposent l’inexistence dans le monde réel d'événements, et plus particulièrement d'états. L’obligation d'états précaires, dans l’introduction des événe- ments des relatives extraposées, est ainsi prise en défaut.

\subsection{Lectures génériques}

Les phrases génériques renvoient, pour faire court, à des ensembles de propriétés/états/événements et d'entités, ou renvoient plutôt à une propriété appliquée à une entité qui parcourt un ensemble de 
mondes possibles. Les constructions DefExtrap dénotent, par contre, des événements-occurrences qui s'inscrivent sous des paramètres d'espace et de temps induits, comme on l'a vu, par les matrices.

DefExtrp est donc exclu des énoncés génériques :

(17) le loup était à l’orée du bois, qui guettait une proie facile

(18) le loup est souvent en bande (Ø+*, qui guette des proies faciles)

L’individu générique le loup de (18) qui se voit attribuer la propriété de vivre souvent en bande, parcourt un ensemble de mondes et n'est instancié dans aucun. L'exigence d'une soumission à des paramètres d'espace et de temps n'est pas satisfaite.

\subsection{Quatre réactifs qui ont vérifié la thèse défendue}

Les quatre réactifs 3.1-3.4 justifient la thèse qui veut que les matrices de DefExtrap aient pour fonction d’installer des états transitoires qui introduisent les événements relatés par les relatives extraposées.

\section{Conclusions}

1. Les constructions qui impliquent des arguments externes modifiés par des relatives extraposées se répartissent, en raison des propriétés qui les particularisent, en deux ensembles. Dans l'un, ces arguments sujets sont définis (DefExtrap) et, dans l'autre, les arguments sont quantifiés (IndefExtrap). Dans ces deux types de constructions, la différence entre défini et quantifieur a ainsi des répercussions étendues.

2. Sous IndefExtrap, contrairement à ce qui est observé sous DefExtrap, la position source (=la trace) du relatif est libre et ce relatif n'est pas réduit à qui sujet, les époques des de propositions ne sont pas contemporaines, le verbe matrice n’est pas nécessairement une copule, le prédicat peut ne pas sélectionner une phase d'individu, mais aussi des individus, c’est-à-dire dénoter des propriétés.

De plus, les matrices de DefExtrap contrastent avec celles de IndefExtrap en ce que, parce qu'elles dénotent des états épisodiques introduisant des événements particuliers, elles ne peuvent pas être niées, être génériques, être contrefactuelles. En effet, rien de tel avec IndefExtrap :

(18) un chat ne dormait pas ici, qui avait, contrairement aux autres, élu domicile au grenier

Un loup part souvent en meute, qui, alors, chasse avec plus d'efficacité

Si des touristes s'étaient perdus à ce moment-là dans le souk, qui auraient vainement cherché leur chemin, je suis sûr qu'Aïda n’aurait pas hésité à les guider vers la sortie

3. Deux questions importantes restent en suspens.

La première concerne le relatif, tête de DefExtrap : comment expliquer la contrainte qui impose à cette position la forme qui (sujet) ? La description sémantique doit manifestement faire place à une analyse syntaxique.

Une piste qui mérite d'être explorée chercherait à attaquer la question par l'autre face : l'exclusive de la position sujet comme source de la tête des relatives extraposées a pour contrepartie l'exclusion de toutes les autres positions. Il s'agit alors d'un cas caractéristique 
d'asymétrie sujets /objets. Les positons gouvernées par $\mathrm{V}^{\circ}$ (le verbe lexical) se verrait barrer la route de la position [Spec,CP] (des relatives) dans une configuration qu'il faut préciser. L'environnement de la position sujet (=[Spec,IP] la prémunirait contre cet accident. La condition de minimalité (Chomsky 1986) révisée par Rizzi 1989 rendrait compte de l'asymétrie sujet/objet en termes de catégories intervenant entre antécédents et positions relativisées.

Une description similaire serait pertinente pour les relatives prédicatives qui présentent la même asymétrie.

Une autre propriété qui réunit DefExtrap et les relatives prédicatives, et qui demandent un éclaircissement, est l'exigence de coïncidence entre les époques des deux propositions engagées.

\section{Références Bibliographiques}

Cecchetto C., 2011. Relabeling Heads. A unified account for relativization structures, Linguistic Inquiry 42, 4. 511-560.

Guasti M.-T., 1988. La pseudo-relative et les phénomènes d'accord, Revista de Grammaica Generativa 13. 35-57.

Kayne R.S., 1977. Syntaxe du français. Le cycle transformationnel du français. 126 et suiv.

Kleiber G., 1988. « Sur les relatives du type je le vois qui arrive », Travaux de linguistique 17. 99-115.

Kupferman L., 2014. "Certains ne dispose-t-il que d’une lecture forte? », Atelier « Les déterminants », Université de Paris-Sorbonne 4, déc. 2014.

Muller C., 2011. «Les interprétations sémantique de la prédication seconde intégrée : les relatives prédicatives », Langue française 171. 101-116.

Prebensen H., 1982. « Les propositions relatives dites ‘attributives’, Revue romane 17. 98-117.

Rothenberg M., 1979. «La proposition relative prédicative et attributive. Problème de linguistique française », Bulletin de la Société de linguistique de Paris 74. 351-395.

Van de Velde D., 2000. "Les indéfinis comme adjectifs", dans De l'indétermination à la qualification : les indéfinis, Arras, Artois Presses Université. 203-272. 